\title{
23. PALEOBIOGEOGRAPHY OF THE PALEOCENE/EOCENE CALCAREOUS NANNOPLANKTON FROM THE NORTH ATLANTIC OCEAN
}

\author{
Bilal U. Haq, Woods Hole Oceanographic Institution, Woods Hole, Massachusetts \\ Hisatake Okada, University of Yamagata, Department of Geology, Yamagata, Japan \\ and \\ G. P. Lohmann, Woods Hole Oceanographic Institution, Woods Hole, Massachusetts
}

\section{INTRODUCTION}

Six sites were cored in the North Atlantic Ocean during Leg 43 of the Deep Sea Drilling Project (DSDP) in June-August 1975 (Figure 1). Of these, three sites $(384,386$, and 397$)$ yielded relatively well preserved Paleocene-Eocene calcareous nannofossil assemblages. At Site 384 an apparently continuous Maestrichtian-Danian transition and a more or less complete Paleocene with well preserved microflora and fauna was recovered (see Okada and Thierstein, this volume, for details of biostratigraphy). During previous attempts at delineating the early Tertiary nannofossil biogeography of the Atlantic Ocean, data from the mid latitudes of the North Atlantic have been relatively scarce (see Haq and Lohmann, 1976). Sites 384, 386 , and 387 cored between $31^{\circ} \mathrm{N}$ and $41^{\circ} \mathrm{N}$ latitude are ideally situated to fill this gap in our knowledge (see Table 1 for summary of coring data for these three sites). This paper is one of a series of special reports written for the Atlantic legs of Phase 3 of 'the DSDP and summarizes the Paleocene-Eocene nannofossil biogeography of the North Atlantic, with special reference to Leg 43 sites. Other reports on early Tertiary paleobiogeography appear in Volumes 36 (Haq et al., 1977), 38 (Haq and Lohmann, 1979) and 39 (Haq et al., 1977).

Our paleobiogeographic method consists of recognizing major and minor assemblages with the help of $Q$-mode varimax factor analysis of the raw census data of relative abundances of taxa. These assemblages are defined in such a way that the constituent taxa contribute most to the samples considered and also approximate the natural taxonomic associations. The details of this method have already been discussed earlier and will not be repeated here (see Haq and Lohmann, 1976, p. 125-134). We have, however, illustrated some of the major biogeographic patterns differently than in the earlier reports. Some of the patterns figured in this paper represents varimax factor loadings (a measure of the abundance of a given assemblage in a sample) as contoured by computer program OPCONT (Cities Service Oil Company). Samples are first plotted according to their age and paleolatitude. Before contouring, OPCONT first generates a regularly spaced grid of the values to be contoured from the original, irregularly spaced, set of samples. The values at the grid in- tersections, the values of the factor loadings which are contoured, are interpolated from nearby samples. The grid we chose to use in this study was spaced at intervals of $1 \mathrm{~m} . \mathrm{y}$. and $10^{\circ}$ paleolatitude.

\section{DISTRIBUTIONAL DATA AND ASSEMBLAGES}

In Tables 2 and 3 we present the distributional data from Paleocene-lower Eocene (NP1-NP12 nannofossil zones) and lower-middle Eocene (NP10-NP15 nannofossil zones), respectively, converted to percentage of relative abundance. Although Leg 43 data contribute only to the early and part of the middle Eocene, the biogeographic patterns summarized here include the entire Eocene of the North Atlantic Ocean.

\section{Paleocene-Lower Eocene}

The census data for Paleocene-lower Eocene interval are presented in Table 2 and light micrographs of most common taxa are illustrated in Plate 1. The biogeographic events for this interval are summarized below:

1) During the earliest Paleocene (early Danian) two assemblages dominate the biogeographic scene in most of the Atlantic Ocean. These are the Thoracosphaerid and the Braarudosphaerid Assemblages (Figure 2). Thoracosphaerids dominate the open ocean sites while braarudosphaerids are the most common taxa in the near-shore and epicontinental areas. The Thoracosphaerid Assemblage as defined by the varimax factor analysis here includes minor amounts of braarudosphaerids and the placoliths of Markalius astroporus. It would thus seem that these two assemblages are essentially similar; the hemipelagic braarudosphaerids are rare in the open ocean and the available niches are overwhelmed by thoracosphaerids. At Site 384 the thoracosphaerids are initially very common but the dominant role is soon assumed by a Cruciplacolithus tenuisZygodiscus Assemblage which ideally consists of equal amounts of these coccolith taxa. This Assemblage remains dominant at Site 384 from about 64.5 to 63 m.y.B.P. (Figure 3).

2) In middle Danian (between 64 and 63 m.y.B.P.) a major coccolith assemblage, the Ericsonia subpertusa Assemblage, first makes its appearance in the lower latitudes and soon becomes important also in the mid latitudes. At Site 384 it becomes important a bit later, around 62 m.y.B.P. This assemblage is composed dom- 


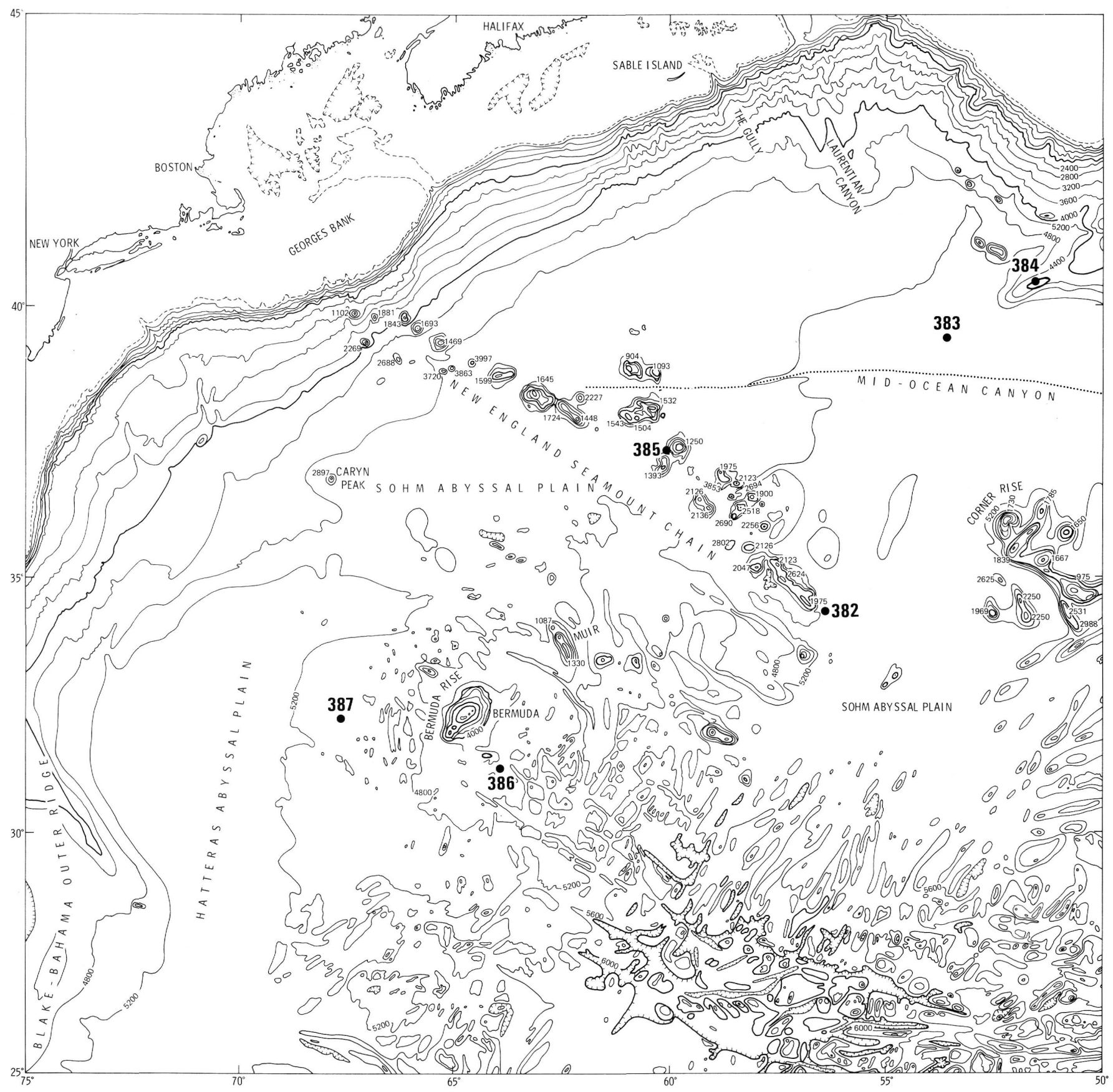

Figure 1. Location of the DSDP Leg 43 drill sites in the North Atlantic.

inantly of E. subpertusa but also contains smaller amounts of Coccolithus pelagicus (s. ampl.); it remains important until 53 m.y.B.P. (late Paleocene), mostly in mid and lower high latitudes (Figure 4).

3) Around 62 m.y.B.P., for a short time, an assemblage composed predominantly of small circular coccoliths (Biscutum ? tenuiculum) becomes important in the middle and lower high latitudes of both the North and South Atlantic (see Figure 5). At Leg 39 Site 356 on the Ceara Rise, southwest Atlantic, the same assemblage (called Prinsius aff. P. dimorphosus in the Leg 39 report, see Haq et al., 1977) was found to be dominant during the same time interval. The occurrence of this assemblage (now described as a new taxon, Biscutum ? tenuiculum; see Okada and Thierstein, this volume) at Site 384 indicates a bimodal and short duration bloom of this taxon in mid to lower high latitudes of the Atlantic.

4) Around 62 m.y.B.P. a relatively high latitude assemblage dominated by the small coccoliths, Prinsius martinii, first occurs in considerable amounts in the high latitudes. At Site 384 it achieves importance a bit later, between 61 and 60 m.y.B.P. The assemblage as a whole shows a latitudinal shift towards mid and low latitudes around 60 m.y.B.P. (Figure 6); it withdraws back to higher latitudes after this short-term invasion 
TABLE 1

Drilling and Coring Summary of Sites 384, 386, and 387

\begin{tabular}{ccccccc}
\hline Site & $\begin{array}{c}\text { Latitude } \\
\text { Longitude }\end{array}$ & $\begin{array}{c}\text { Water } \\
\text { Depth } \\
(\mathrm{m})\end{array}$ & $\begin{array}{c}\text { Meters } \\
\text { Cored }\end{array}$ & $\begin{array}{c}\text { Meters } \\
\text { Recovered }\end{array}$ & $\begin{array}{c}\text { Oldest } \\
\text { Sediment }\end{array}$ & $\begin{array}{c}\text { Intervals Considered For } \\
\text { Paleobiogeography } \\
\text { (Nannozones) }\end{array}$ \\
\hline 384 & $\begin{array}{l}40^{\circ} 21.65^{\prime} \mathrm{N} \\
51^{\circ} 39.80^{\prime} \mathrm{W}\end{array}$ & 3910 & 195.3 & 110.5 & $\begin{array}{l}\text { Aptian or } \\
\text { older }\end{array}$ & NP1-NP9, NP14 \\
386 & $\begin{array}{l}31^{\circ} 11.21^{\prime} \mathrm{N} \\
64^{\circ} 14.94^{\prime} \mathrm{W}\end{array}$ & 4783 & 626.7 & 438.2 & $\begin{array}{l}\text { Upper } \\
\text { Aptian- } \\
\text { lower } \\
\text { Albian }\end{array}$ & NP7, NP9-NP15 \\
387 & $\begin{array}{l}32^{\circ} 19.2^{\prime} \mathrm{N} \\
67^{\circ} 40^{\prime} \mathrm{W}\end{array}$ & 5118 & 467.9 & 178.2 & $\begin{array}{l}\text { Upper } \\
\text { Berriasian- } \\
\text { lower } \\
\text { Valanginian }\end{array}$ & NP12-NP15 \\
\hline
\end{tabular}

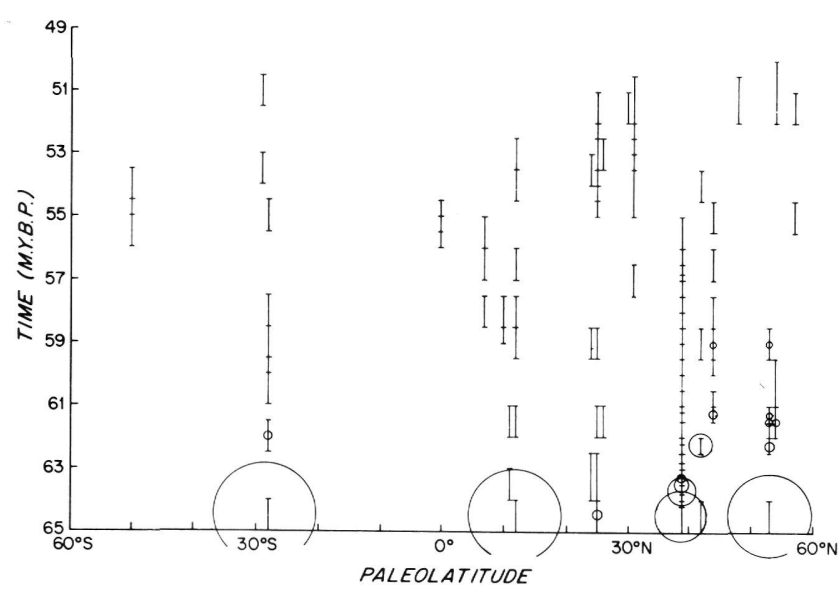

A

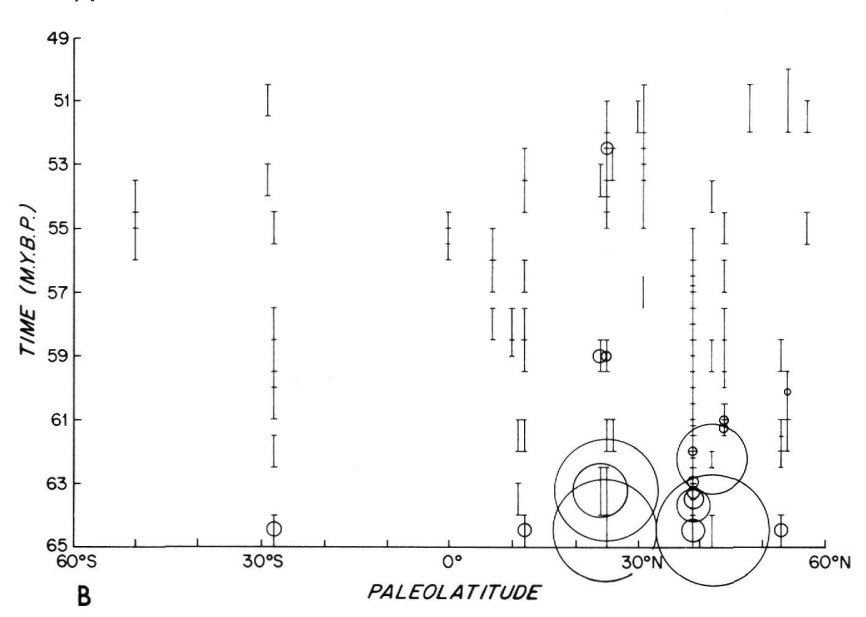

Figure 2. Time and paleolatitudinal distribution of the Thoracosphaerid (A) and the Braarudosphaerid (B) assemblages of the early Paleocene. The length of the bar along each sample represents the uncertainty of the age of the sample, and the size of the circle around it is proportional to a measure of the relative abundance (factor loading) of the assemblage in that sample (see Haq and Lohmann, 1976).

of the lower latitudes. Another minor assemblage (Prinsius bisulcus), similar in latitudinal preference to $P$. martinii, becomes relatively important at Site 384 at

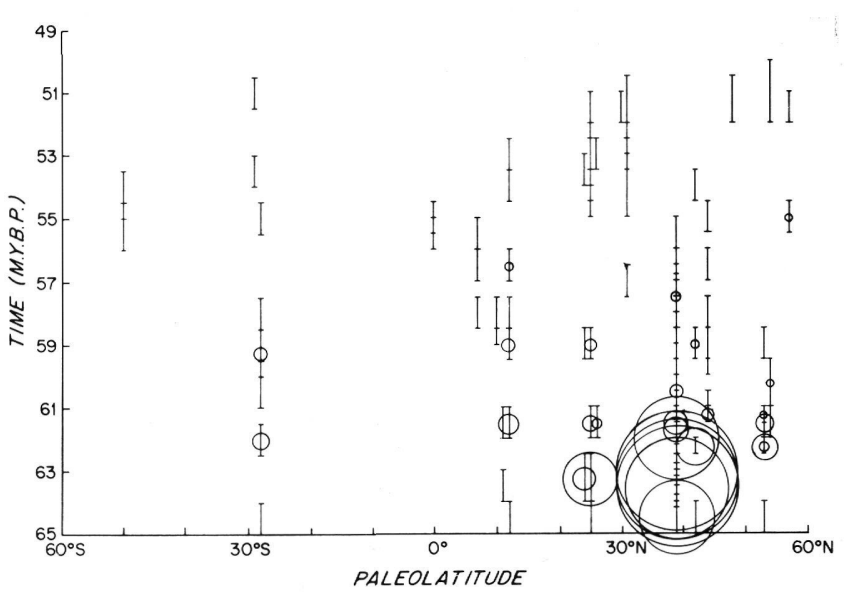

Figure 3. The Cruciplacolithus tenuis-Zygodiscus Assemblage of the early Paleocene. This is particularly abundant at Site 384 between 64.5 and 63 m.y.B.P.

about 60 m.y.B.P. Elsewhere this assemblage occurs in minor amounts (see Figure 7).

5) The last major assemblage of the Paleocene-early Eocene interval is composed predominantly of the small coccoliths Toweius craticulus and minor amounts of Coccolithus pelagicus ( $s$. ampl.). It first appears around 63 m.y.B.P., becoming important at Site 384 around 61 m.y.B.P. and then spreading from mid to low latitudes around 59 m.y.B.P. It shows an important shift into higher latitudes in the late Paleocene-early Eocene (see Figure 8). It is also an important component of the nannoflora at Site 386 in the late Paleocene-early Eocene (NP9-NP12) interval.

\section{Eocene}

The Eocene census data are presented in Table 3 and light micrographs of most common Eocene taxa are illustrated in Plate 2.

Five major assemblages are recognized in the Eocene interval. Of these only four are important at Sites 384,386 , and 387 in the early to middle Eocene interval considered here.

1) During the early Eocene the Paleocene Towieus craticulus Assemblage continues to be important in low to mid latitudes, showing a shift to higher latitudes be- 
TABLE 2

Paleocene-Early Eocene (NP1-NP12 Nannoplankton Zones) Census Data Converted to Percentage of Total from DSDP Sites 384 and 386

\begin{tabular}{|c|c|c|c|c|c|c|c|c|c|c|c|c|c|c|c|c|c|c|c|c|c|c|c|c|}
\hline $\begin{array}{c}\text { Sample } \\
\text { (top cm only) }\end{array}$ & : & 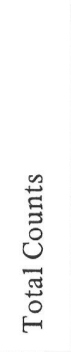 & 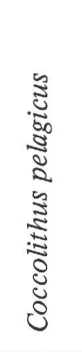 & 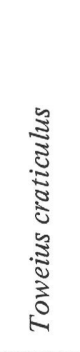 & 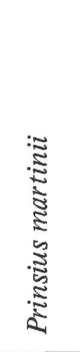 & 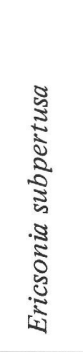 & 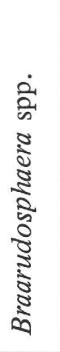 & 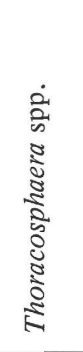 & 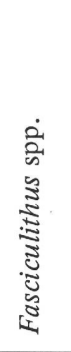 & 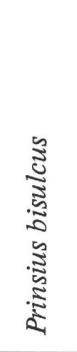 & 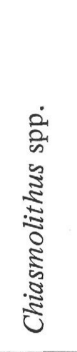 & 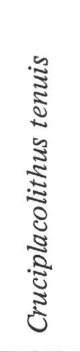 & 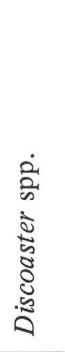 & 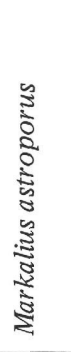 & 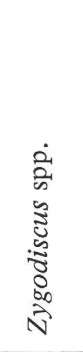 & 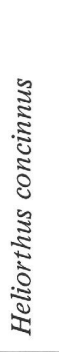 & 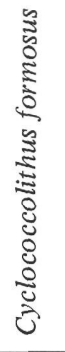 & 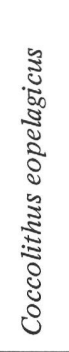 & 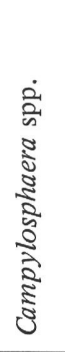 & 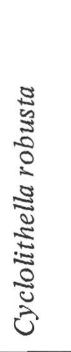 & 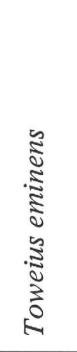 & 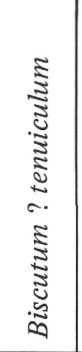 & 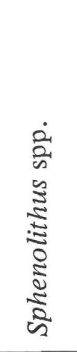 & 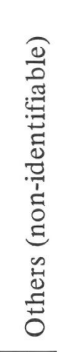 \\
\hline $384-13-3,32$ & NP1 & 318 & 0.0 & 0.0 & 0.0 & 0.0 & 4.4 & 17.0 & 0.0 & 0.0 & 0.0 & 20.8 & 0.0 & 8.5 & 34.9 & 0.0 & 0.0 & 0.0 & 0.0 & 0.0 & 0.0 & 0.0 & 0.0 & 14.5 \\
\hline $7-13-3,8$ & NP2 & 306 & 0.0 & 0.0 & 0 . & 0.0 & 9.8 & 10.5 & 0.0 & 0.0 & 0.0 & 40.2 & 0. & 6.2 & 32.4 & 0.0 & 0.0 & 0.0 & 0 . & 0 & 0.0 & 0.0 & 0.0 & 1.0 \\
\hline $384-13-2,134$ & NP2 & 305 & 0.0 & 0.0 & 0.0 & 0.0 & 4.3 & 5.6 & 0.0 & 0.0 & 0.0 & 73.1 & $0 .($ & 4.3 & 12.1 & 0 . & 0.0 & 0.0 & 0 . & & 0.0 & 0.0 & 0.0 & 0.7 \\
\hline $384-13-2,80$ & NP2 & 306 & 0.0 & 0.0 & 0.0 & 0.0 & 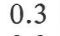 & 2.6 & 0.0 & 0.0 & 0.0 & 92.8 & $0 .($ & 0.3 & 3.6 & 0.0 & 0.0 & 0.0 & 0.0 & 0.0 & 0.0 & 0.0 & 0.0 & 0.3 \\
\hline $384-13-1,145$ & NP2 & 308 & 17.5 & 0.0 & 0.3 & 4.2 & 0.0 & 1.0 & 0.0 & 0.0 & 0.0 & 72.4 & 0.0 & 0.3 & 2.6 & 0.0 & 0.0 & 0.0 & 0.0 & 0.0 & 0.0 & 0.3 & 0.0 & 1.3 \\
\hline $384-12-6,50$ & NP3 & 309 & 20.7 & 0.0 & 0.3 & 10.7 & 0.0 & 0.3 & 0.0 & 0.0 & 0.3 & 32.7 & 0.0 & 0.3 & 8.4 & 0.0 & 0.0 & 0.0 & 0.0 & 0.0 & 0.0 & 25.9 & 0.0 & 0.3 \\
\hline $384-12-4,120$ & NP3 & 299 & 31.4 & 0.0 & 1.0 & 3.7 & 0. & 0.0 & 0.0 & 0.0 & 3.3 & 11.7 & 0.0 & 0.3 & 1.3 & 0.0 & 0.0 & 0.0 & 0.0 & 0.0 & 0.0 & 47.2 & 0.0 & 0.0 \\
\hline $384-12-2,110$ & NP3 & 305 & 42.3 & 0.0 & 3.6 & 30.5 & $0 .($ & 0.0 & 0.0 & 0.7 & 2.3 & 11.5 & $0 .($ & 0.0 & 2.6 & 0.0 & 0.0 & 0.0 & 0.0 & 0.0 & 0.0 & 4.3 & 0.0 & 2.3 \\
\hline $1, \mathrm{CC}$ & NP4 & 307 & 25.1 & 0.0 & 29.6 & 19.5 & 0. & 0.3 & 0.0 & 9.4 & 2.6 & 7.5 & $0 .($ & 0.3 & 2.9 & 0. & 0.0 & 0.0 & 0.0 & 0.0 & 0.0 & 0.7 & 0.0 & 2.0 \\
\hline$-6,60$ & NP4 & 325 & 21.2 & 7.4 & 18.5 & 6.8 & 0. & 0.3 & 0.0 & 25.8 & 4.9 & 3.4 & 0.0 & 0.3 & 3.1 & 0. & 0.0 & 0.0 & 0.0 & 0.0 & 0.0 & 0.0 & 4.3 & 4.0 \\
\hline $384-10-2,120$ & NP5 & 301 & 36.5 & 7.0 & 7.6 & 9.3 & 0.0 & 0.3 & 0.0 & 9.6 & 4.3 & 1.7 & 0.0 & 0.7 & 2.7 & 4.7 & 0.0 & 0.0 & 0.0 & 0.3 & 0.0 & 0.0 & 12.3 & 3.0 \\
\hline & NP5 & 304 & 35.2 & 9.2 & 5.6 & 11.5 & 0.0 & 0.3 & 3.0 & 5.6 & 76 & 0.7 & 0. & 0.0 & 3.9 & 0.0 & 0.0 & 0.0 & 0.0 & 0 . & 1.0 & 0.0 & 14.8 & 1.6 \\
\hline$-1,125$ & P5 & 300 & 32.3 & 3.3 & 2.3 & 15.3 & 0 . & 0.0 & 0.0 & 8.3 & 10.7 & 0.0 & 0. & 0.0 & 9. & 0 & 0.0 & 0.0 & 5.7 & 0.0 & 5.0 & 0.0 & 7.3 & 0.7 \\
\hline 4,50 & NP6 & 300 & 27.7 & 7.7 & 2.7 & 11.3 & 0. & 0.0 & 7.3 & 5.3 & 8.0 & 1.3 & 0.3 & 0.0 & 7.7 & 1.3 & 0.0 & 0.0 & 0.0 & 0.0 & 4.3 & 0.0 & 5.7 & 9.3 \\
\hline $384-7-6,110$ & NP7 & 303 & 41.6 & 5.9 & 1.0 & 6.3 & 0.0 & 0.0 & 7.3 & 6.3 & 7.9 & 1.0 & 4.0 & 0.0 & 8.3 & 0.0 & 0.0 & 0.0 & 0.0 & 0.0 & 4.3 & 0.0 & 4.0 & 2.3 \\
\hline 125 & NP7 & 304 & 37.5 & 4.9 & 3.0 & 10.2 & 0.0 & 0.0 & 6.6 & 3.9 & 7.2 & 1.0 & 1.6 & 0.0 & 12.8 & 0.0 & 0.0 & 0.0 & 0.0 & 0.3 & 2.3 & 0.0 & 4.9 & 3.6 \\
\hline 1,40 & 7 & 3 & 49.7 & 4. & 0. & 13 & 0 & 0. & 8. & 2 & 3. & 0 . & 1 & 0. & 6 & 0 . & 0.0 & 0. & 0 & 0 . & 0.6 & 0.0 & 1.9 & 6.1 \\
\hline $384-7-4,50$ & NP8 & 302 & 31.5 & 7.6 & 3.0 & 7.6 & 0. & 0.3 & 5.0 & 8.5 & 7.3 & 0.3 & 5. & 0.0 & 8.9 & 0 . & 0.0 & 0.0 & 0.0 & 0 & 5.0 & 0.0 & 7.3 & 1.7 \\
\hline$-4,110$ & NP8 & 302 & 40.1 & 7.9 & 1.7 & 9.3 & 0.0 & 0.0 & 8.6 & 6.3 & 3.3 & 0.0 & $5 .($ & 0.0 & 1.0 & 0.0 & 0.0 & 0.0 & 0.0 & 2.3 & 2.3 & 0.0 & 9.3 & 3.0 \\
\hline$-2,40$ & NP8 & 302 & 42.1 & 7.0 & 2.6 & 6.3 & 0.0 & 0.0 & 6.3 & 4.6 & 4.3 & 0.0 & 1.0 & 0.0 & 0.3 & 0.0 & 0.0 & 0.0 & 0.0 & 2.0 & 7.9 & 0.0 & 10.9 & 4.6 \\
\hline$-1,75$ & NP9 & 303 & 31.7 & 6.9 & 1.0 & 4.6 & 0.0 & 0.0 & 12.5 & 5.6 & 4.6 & 0.3 & 4.3 & 0.7 & 0.0 & 0.0 & 0.0 & 0.0 & 0.0 & 3.3 & 8.3 & 0.0 & 13.2 & 3.0 \\
\hline $384-6-1,15$ & NP9 & 303 & 33.0 & 12.9 & 3.0 & 1.0 & 0.0 & 0.0 & 12.2 & 6.3 & 3.3 & 0.0 & 5.0 & 0.0 & 1.0 & 0.0 & 0.0 & 0.0 & 0.0 & 0.0 & 12.5 & 0.0 & 7.9 & 2.0 \\
\hline $386-34-3,82$ & NP9 & 301 & 31.6 & 25.2 & 0.0 & 7.0 & 0.0 & 0.0 & 7.6 & 11.6 & 0.0 & 0.7 & 3.0 & 0.0 & 1.7 & 0.0 & 0.0 & 3.0 & 0.3 & 0.7 & 5.3 & 0.0 & 0.3 & 2.0 \\
\hline $3-3,82$ & NP10 & 323 & 30.0 & 39.3 & 0 . & 5.3 & 0. & 0.0 & 0.0 & 5.6 & 1.5 & 0.0 & 7. & 0.0 & 0. & 0 . & 2.2 & 3.1 & 0.0 & 0.0 & 0.0 & 0.0 & 3.7 & 2.2 \\
\hline $386-34-1,78$ & NP10 & 328 & 37.5 & 22.9 & 0.0 & 4.9 & 0.0 & 0.0 & 2.1 & 6.7 & 1.8 & 0.0 & 4.6 & 0.0 & 0.0 & 0.0 & 3.5 & 5.8 & 0.3 & 0.0 & 9.8 & 0.0 & 0.3 & 3.4 \\
\hline $386-33-2,50$ & NP11 & 320 & 15.6 & 35.3 & 14.1 & 0.9 & 0.0 & 0.0 & 0.0 & 5.3 & 2.8 & 0.0 & 4.7 & 0.0 & 0.0 & 0.0 & 1.9 & 4.4 & 1.9 & 0.0 & 0.0 & 0.0 & 5.6 & 7.5 \\
\hline & & 31 & 24.4 & 39.4 & 4.8 & 0 . & 0 & 0.3 & 0 . & 2. & 1. & 0. & 4. & 0 . & 0. & 0 & 3.8 & 1. & & & & 0.0 & 38 & 11.5 \\
\hline $386-31-3,85$ & NP12 & 327 & 28.1 & 34.3 & 3.4 & 0.0 & 0.0 & 0.0 & 0.0 & 3.4 & 2.1 & 0.0 & 8.3 & 0.0 & 0.0 & 0.0 & 4.0 & 1.2 & 0.9 & 0.0 & 0.0 & 0.0 & 2.8 & 11.6 \\
\hline
\end{tabular}


TABLE 3

Eocene (NP10-NP15 Zones) Census Data Converted to Percentage of Total From DSDP Leg 43 Sites 384, 386, and 387 (for zone designations see Okada and Thierstein, this volume)

\begin{tabular}{|c|c|c|c|c|c|c|c|c|c|c|c|c|c|c|c|c|c|c|c|c|c|c|}
\hline $\begin{array}{c}\text { Sample } \\
\text { (top cm only) }\end{array}$ & 芯 & 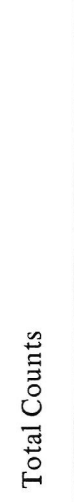 & 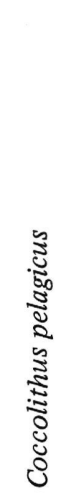 & 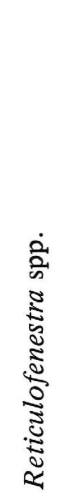 & 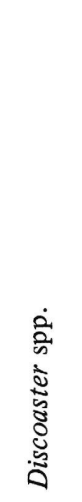 & 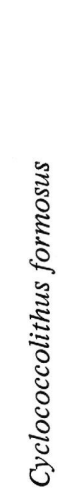 & 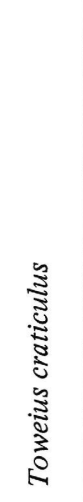 & 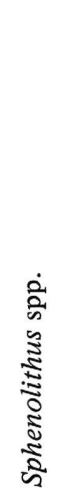 & 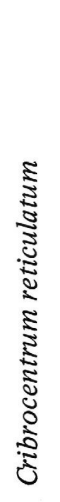 & 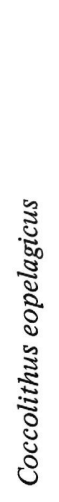 & 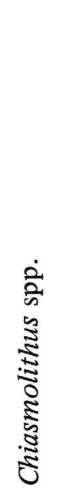 & 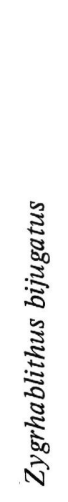 & 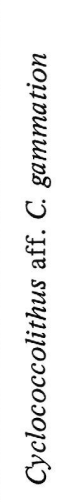 & 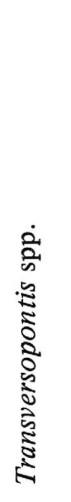 & 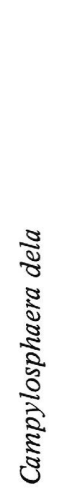 & 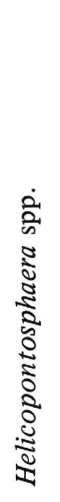 & 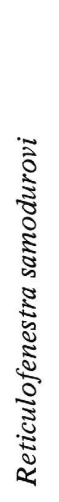 & 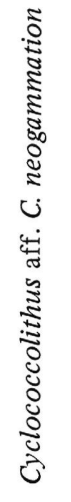 & 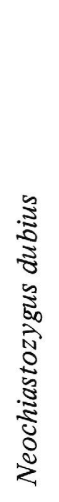 & 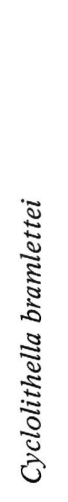 & 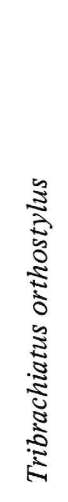 & 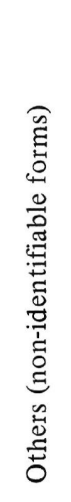 \\
\hline $386-34-1,78$ & NP10 & 328 & 37.5 & 0.0 & 4.6 & 0.0 & 22.9 & 0.3 & 0.0 & 5.8 & 1.8 & 0.0 & 0.0 & 0.0 & 0.0 & 0.0 & 0.0 & 0.0 & 0.0 & 0.0 & 0.0 & 27.1 \\
\hline $6-33-3,82$ & NP10 & 323 & 30.0 & 0.0 & 7.1 & 2.2 & 39.3 & 3.7 & 0.0 & 3.1 & 1.5 & 0.0 & 0.0 & 0. & 0.0 & 0.0 & 0.0 & 0.0 & 0.0 & 0 . & 0.0 & 12.7 \\
\hline $386-33-2,50$ & NP11 & 320 & 15.6 & 0.0 & 4.7 & 1.9 & 35.3 & 5.6 & 0.0 & 4.4 & 2.8 & 0.6 & 0.0 & 0.3 & 1.9 & 0.0 & 0.0 & 0.0 & 0.3 & 0.3 & 1.6 & 24.7 \\
\hline $386-31-3,85$ & NP12 & 327 & 28.1 & 1.2 & 8.3 & 4.0 & 34.3 & 2.8 & 0.0 & 1.2 & 2.1 & 2.1 & 0.6 & 0.6 & 0.9 & 0.0 & 0.0 & 0.0 & 0.9 & 0.3 & 1.2 & 11.3 \\
\hline $386-32-4,63$ & NP12 & 312 & 24.4 & 0.6 & 4.2 & 3.8 & 39.4 & 3.8 & 0.0 & 1.6 & 1.3 & 3.2 & 0.0 & 1.0 & 1.3 & 0.0 & 0.0 & 0.0 & 1.0 & 0.0 & 1.0 & 13.4 \\
\hline $387-23-2,107$ & NP12 & 312 & 22.4 & 27.5 & 8.0 & 3.2 & 3.2 & 3.8 & 0.0 & 3.2 & 4.5 & 1.0 & 0.3 & 0.6 & 3.5 & 0.0 & 0.0 & 0.0 & 1.0 & 0.3 & 1.6 & 15.7 \\
\hline $387-22-2,30$ & NP12 & 314 & 29.3 & 18.5 & 3.2 & 3.2 & 7.0 & 2.2 & 0.0 & 5.7 & 3.5 & 1. & 0.0 & 0.0 & 1.6 & 0.0 & 0.0 & 0.0 & 0.6 & 1.0 & 0.6 & 21.7 \\
\hline $386-30-4,100$ & NP13 & 336 & 36.3 & 0.6 & 15.8 & 10.1 & 12.2 & 1.5 & 0.0 & 5.7 & 3.0 & 7.7 & 2.4 & 0.6 & 2.1 & 0.0 & 0.0 & 0.0 & 0.6 & 0.0 & 0.0 & 1.5 \\
\hline $387-21, \mathrm{CC}$ & NP13 & 322 & 29.5 & 18.0 & 7.5 & 8.1 & 1.9 & 3.4 & 0.3 & 9.9 & 6.2 & 2.5 & 1.2 & 0.0 & 4.0 & 0.0 & 0.0 & 0.0 & 0.9 & 0.6 & 0.3 & 5.6 \\
\hline $384-5-2,55$ & NP14A & 301 & 13.3 & 40.2 & 4.7 & 6.6 & 0.0 & 6.3 & 0.0 & 4.0 & 6.0 & 0.7 & 1.0 & 0.0 & 0.0 & 1.0 & 0.7 & 6.6 & 0.0 & 0.3 & 0.0 & 8.6 \\
\hline $386-29-2,60$ & NP14A & 330 & 22.4 & 19.4 & 12.4 & 9.7 & 6.7 & 3.0 & 0.3 & 4.2 & 5.8 & 3.0 & 1.5 & 2.1 & 2.7 & 0.6 & 0.0 & 2.7 & 0.9 & 0.0 & 0.0 & 2.4 \\
\hline $387-20-1,120$ & NP14A & 318 & 26.7 & 26.7 & 7.9 & 2.2 & 4.1 & 0.9 & 0.3 & 4.1 & 5.3 & 2.2 & 0.9 & 3.8 & 1.3 & 1.9 & 2.8 & 0.9 & 1.3 & 0.3 & 0.0 & 6.3 \\
\hline $384-4-6,144$ & NP14B & 306 & 12.4 & 37.6 & 10.5 & 8.5 & 0.0 & 2.6 & 0.0 & 7.2 & 5.9 & 0.3 & 0.7 & 0.0 & 0.0 & 0.7 & 1.6 & 8.2 & 0.0 & 1.3 & 0.0 & 2.6 \\
\hline $386-27-4,118$ & NP14B & 342 & 20.2 & 37.1 & 11.1 & 9.4 & 0.9 & 0.9 & 0.0 & 3.8 & 4.4 & 1.8 & 0.3 & 0.9 & 0.6 & 1.2 & 2.6 & 1.2 & 0.3 & 0.3 & 0.0 & 3.2 \\
\hline $384-4-3,50$ & NP15A & 306 & 16.7 & 31.4 & 8.2 & 7.8 & 0.0 & 3.3 & 0.7 & 2.9 & 10.5 & 4.6 & 0.7 & 0.0 & 0.3 & 0.3 & 2.6 & 3.9 & 0.0 & 1.3 & 0.0 & 4.9 \\
\hline $386-23-5,100$ & NP15A & 342 & 12.0 & 41.8 & 13.2 & 7.3 & 0.3 & 1.2 & 0.0 & 1.8 & 6.7 & 1.2 & 0.0 & 1.5 & 0.6 & 0.9 & 4.1 & 4.1 & 0.3 & 0.0 & 0.0 & 3.2 \\
\hline $387-16-2,20$ & $15 \mathrm{~A}$ & 308 & 11.4 & 52.6 & 0.6 & 7.1 & 0.6 & 0.3 & 0.0 & 1.3 & 3.9 & 0.6 & 0.0 & 7.5 & 0.3 & 0.6 & 3.9 & 0.3 & 1.6 & 1.3 & 0.0 & 5.8 \\
\hline $384-2-2,50$ & NP15B & 311 & 15.1 & 36.7 & 6.1 & 10.3 & 0.0 & 4.2 & 0.6 & 4.8 & 4.8 & 0.3 & 0.6 & 0.0 & 0.3 & 0.3 & 7.7 & 2.9 & 0.0 & 0.6 & 0.0 & 4.5 \\
\hline $387-12-1,120$ & NP15B & 306 & 10.1 & 45.8 & 2.3 & 6.2 & 1.3 & 0.3 & 0.0 & 1.3 & 13.4 & 0.3 & 0.0 & 7.2 & 0.0 & 0.0 & 2.9 & 0.7 & 1.0 & 0.3 & 0.0 & 6.9 \\
\hline $384-1-1,110$ & NP15C & 309 & 19.4 & 33.7 & 3.2 & 11.0 & 0.0 & 4.2 & 0.6 & 8.4 & 3.9 & 0. & 0.0 & 0.0 & 0.6 & 1.0 & 3.9 & 4.9 & 0.0 & 0.6 & 0.0 & 4.5 \\
\hline $387-10-3,60$ & NP15C & 308 & 7.5 & 71.1 & 2.6 & 6.2 & 0.6 & 0.6 & 0.0 & 0.3 & 1.9 & 0.6 & 0.0 & 0.3 & 0.6 & 0.0 & 2.3 & 0.3 & 0.3 & 0.3 & 0.0 & 4.2 \\
\hline $387-9-1,100$ & NP15C & 310 & 3.2 & 63.9 & 1.3 & 8.1 & 0.3 & 2.6 & 0.0 & 1.6 & 2.6 & 2.3 & 0.0 & 1.9 & 0.3 & 1.9 & 2.9 & 0.6 & 2.3 & 0.0 & 0.0 & 4.2 \\
\hline
\end{tabular}




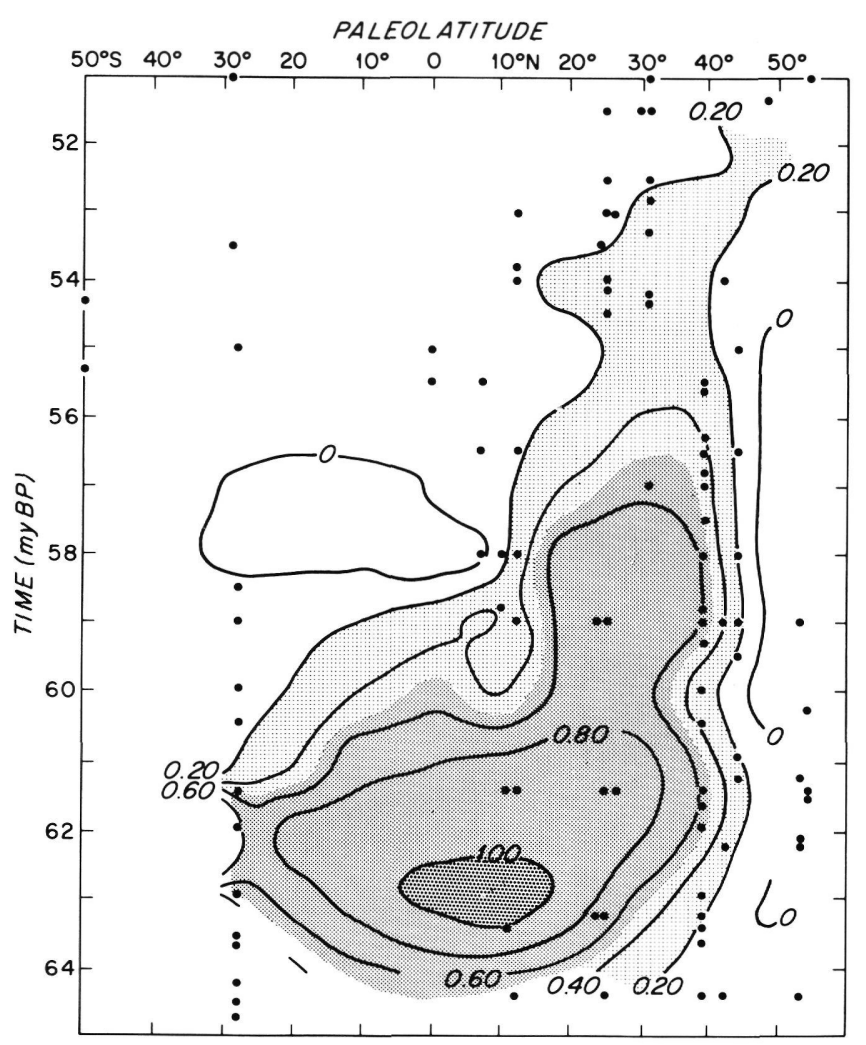

Figure 4. Time and paleolatitudinal distribution of the Paleocene Ericsonia subpertusa Assemblage (for an explanation of contouring method see text). Heaviness of the shaded areas is proportional to the relative abundance (factor loadings) of the assemblage.

tween 52 to 50 m.y.B.P. At Site 386 it remains relatively important until 50 m.y.B.P. (NP13 Zone). After 49 m.y.B.P. it loses its prominence at all latitudes (Figure 9).

2) A new assemblage composed of small to medium sized reticulofenestrids appears in the later part of the early Eocene, occupying the niche vacated by the Toweius craticulus Assemblage. This is a relatively mid latitude assemblage which spreads into lower and higher latitudes between 50 and 49 m.y.B.P. and then becomes most important in mid latitudes until 45 m.y.B.P. when it shows a shift toward lower latitudes. It is important in the mid Eocene of all Leg 43 sites considered here (Figure 10).

3 ) Another group of taxa that achieve importance in the Eocene are the discoasters. The Discoaster Assemblage first achieves prominence between 52 and 51 m.y.B.P. in the low latitudes, then spreading into mid latitudes by 50 m.y.B.P. It withdraws to low latitudes between 47 and 45 m.y.B.P. and once again spreads in mid and even higher latitudes between 45 and 43 m.y.B.P. It loses importance after 43 m.y.B.P. (Figure 11). At Leg 43 sites it is relatively important around 49 m.y.B.P. (NP14 and NP15 zones).

4) The fourth assemblage is dominated by Coccolithus pelagicus ( $s$. ampl.) with smaller amounts of $C y$ clococcolithus formosus. Its main distribution is in the

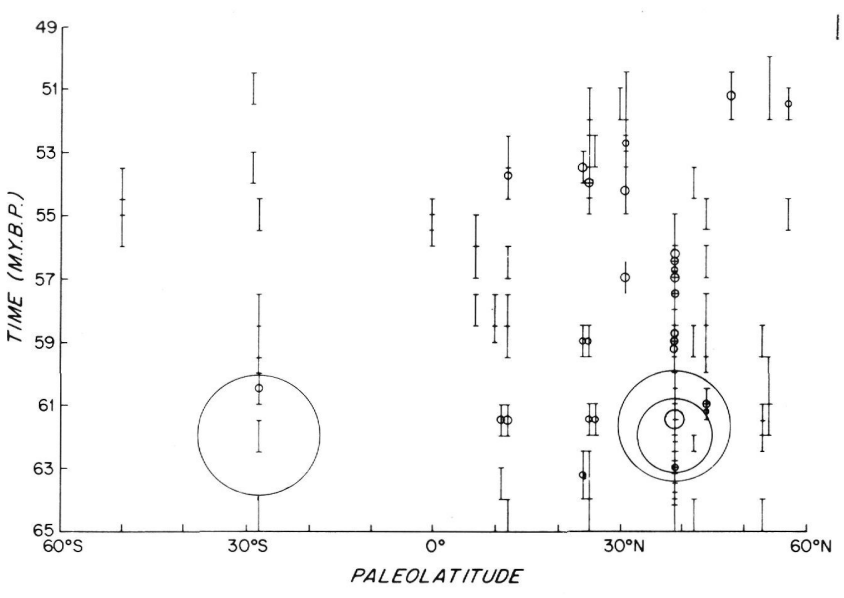

Figure 5. The short-term bimodal prominence of the Biscutum? tenuiculum Assemblage lat Site 384 in the North and Site 356 in the South Atlantic).

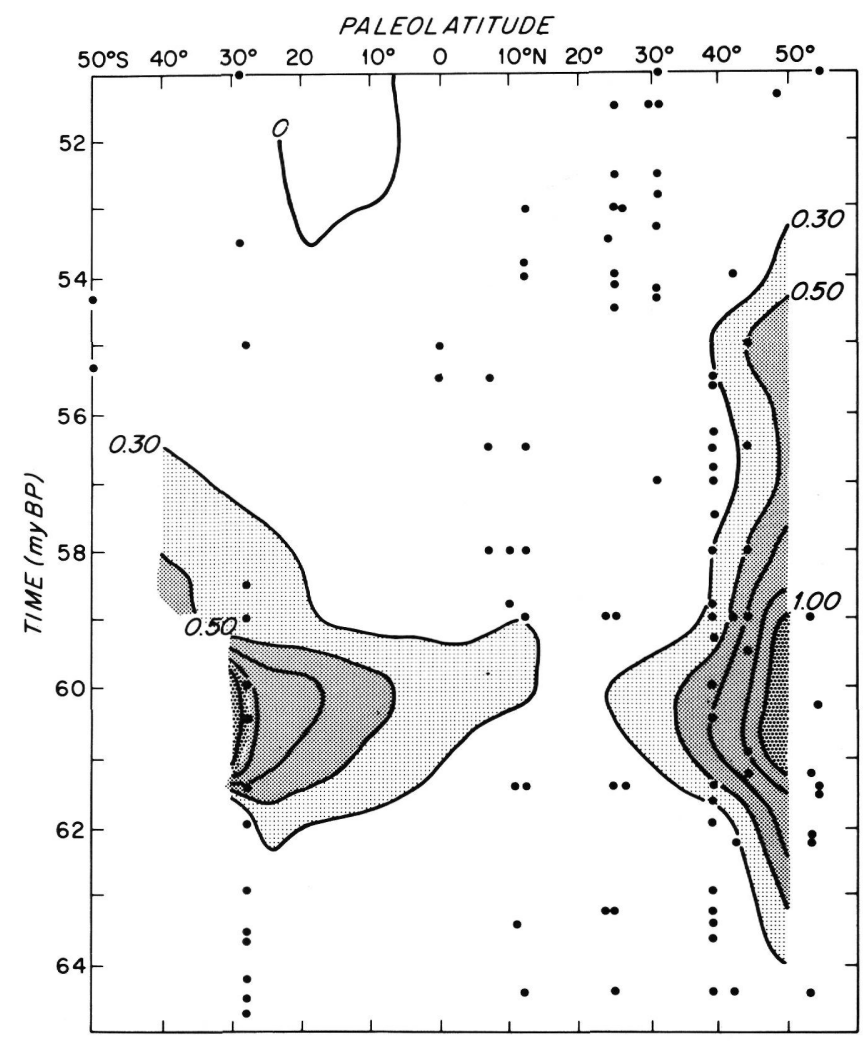

Figure 6. Time and paleolatitudinal distribution of the relatively high latitude Prinsius martinii Assemblage of the Paleocene.

mid latitudes, shifting slightly towards lower latitudes in the later part of middle Eocene. At Sites 386 and 387 it is relatively important through all of the interval considered here (Figure 12).

5) The last major assemblage of the Eocene (Reticulofenestra umbilica) is not important in the early to middle Eocene interval of Leg 43 sites considered here. Elsewhere it becomes important in the later part of middle Eocene and late Eocene. 


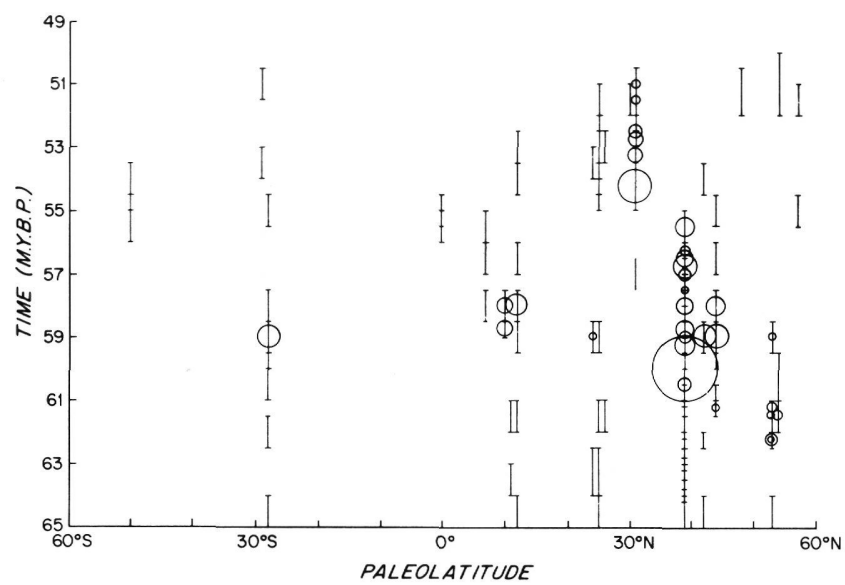

Figure 7. Distribution of the relatively minor Prinsius bisulcus Assemblage.

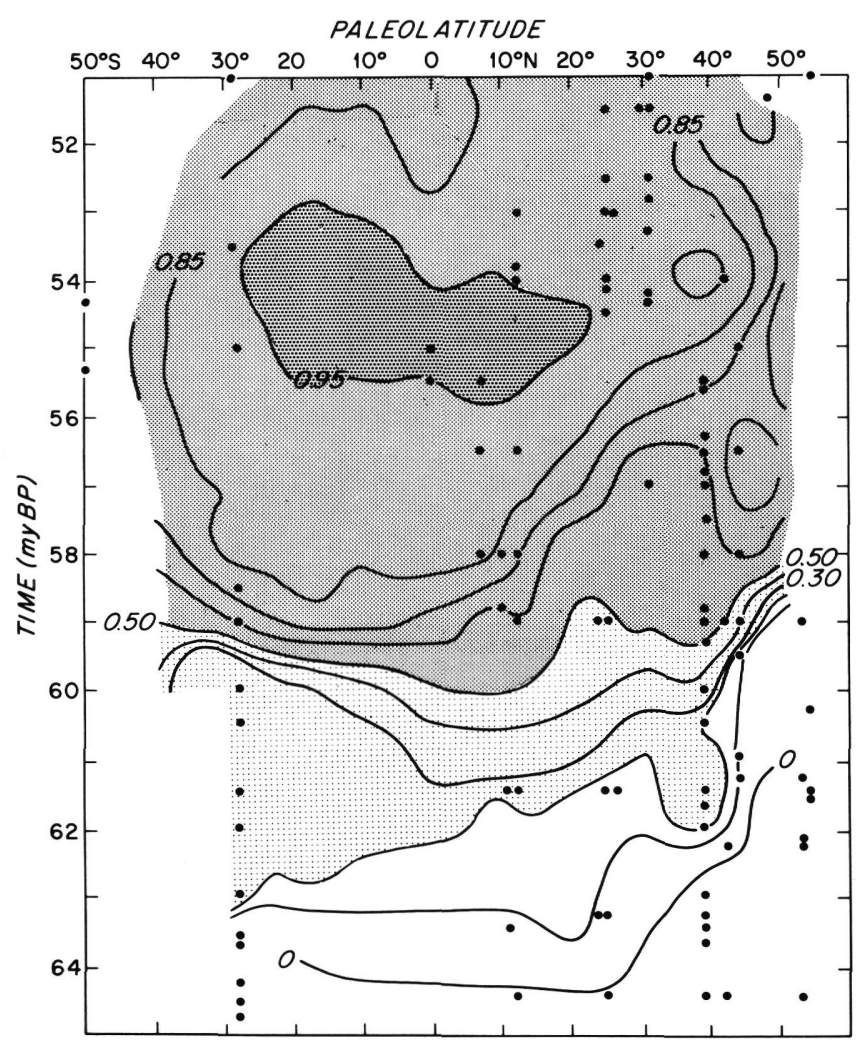

Figure 8. Time and paleolatitudinal distribution of the Toweius craticulus Assemblage during the Paleoceneearly Eocene interval.

\section{PALEOCLIMATIC IMPLICATIONS}

By analogy with the present-day climatically differentiated nannoplankton assemblages, Haq and Lohmann (1976) attributed the early Cenozoic latitudinal differentiations to climatic control. After classifying all assemblages along a relative temperature gradient by comparing them with latitudinally restricted "indicator" assemblages, they interpreted the major shifts in the distributional patterns of these assemblages to be

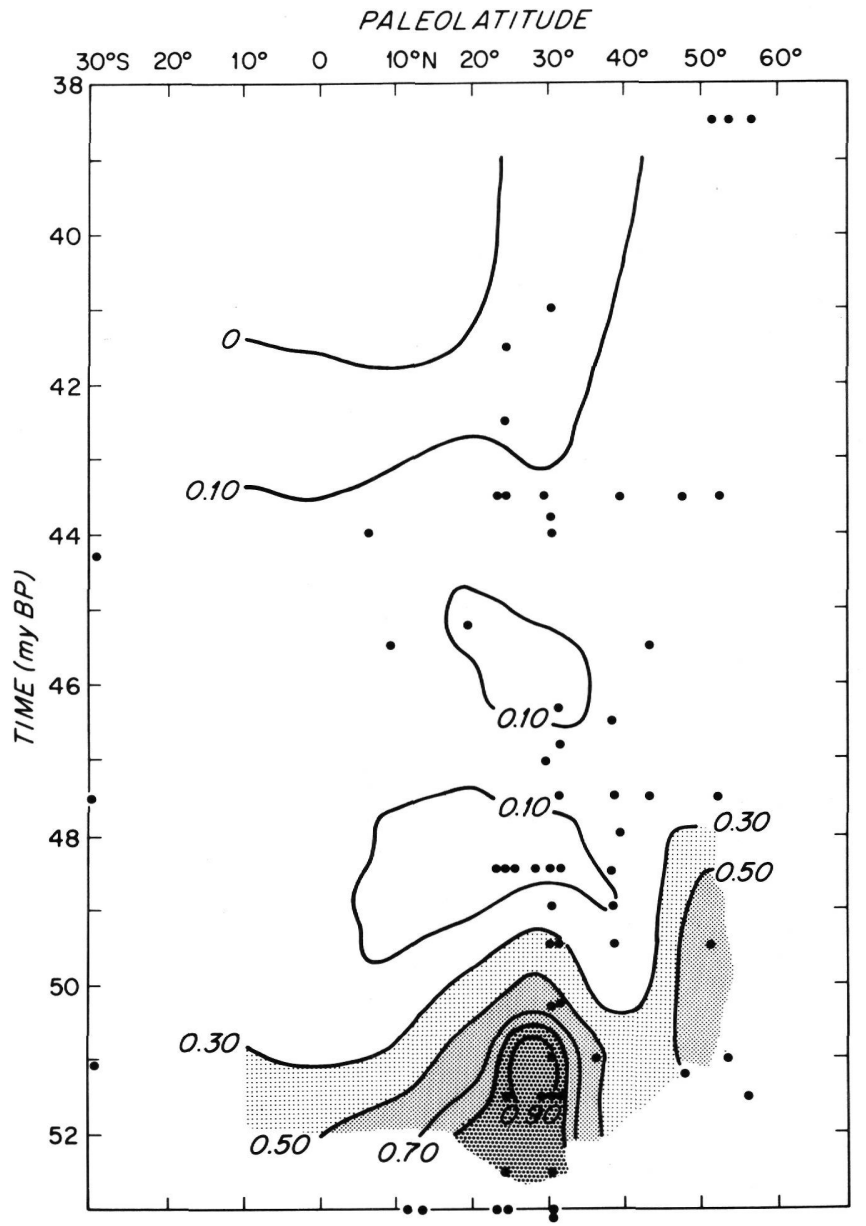

Figure 9. Time and paleolatitudinal distribution of the Toweius craticulus Assemblage during the Eocene.

caused by major fluctuations in the surface water temperatures of the ocean. They inferred two marked cooling episodes during the Paleocene-Eocene interval, the first during middle Paleocene and the second during middle Eocene; a marked warming event during the late Paleocene and early Eocene, and a second, less pronounced warming in the late Eocene.

With the additional data from Leg 43 and the patterns as they are presented here, it is now possible to define more accurately the durations of these climatic episodes. (1) The middle Paleocene cooling episode can now be dated to have occurred between 61 and 58 m.y.B.P., with a peak around 60 m.y.B.P. as shown by the migratory patterns of the high latitude Prinsius martinii Assemblage (see Figure 8). (2) The late Paleocene-early Eocene warming episode occurred between 54 and 48 m.y.B.P. with peak warming between 50 and 49 m.y.B.P. when the warm Toweius craticulus Assemblage shows its maximum poleward extent (see Figures 8,9). (3) The middle Eocene cooling occurred between 48 and 45 m.y.B.P. when discoasters retreat from relatively mid to high latitudes towards low latitudes. Particularly, between 47 and 45 m.y.B.P. they become restricted to low latitudes. A slight withdrawal of the Reticulofenestrid Assemblage from higher lati- 


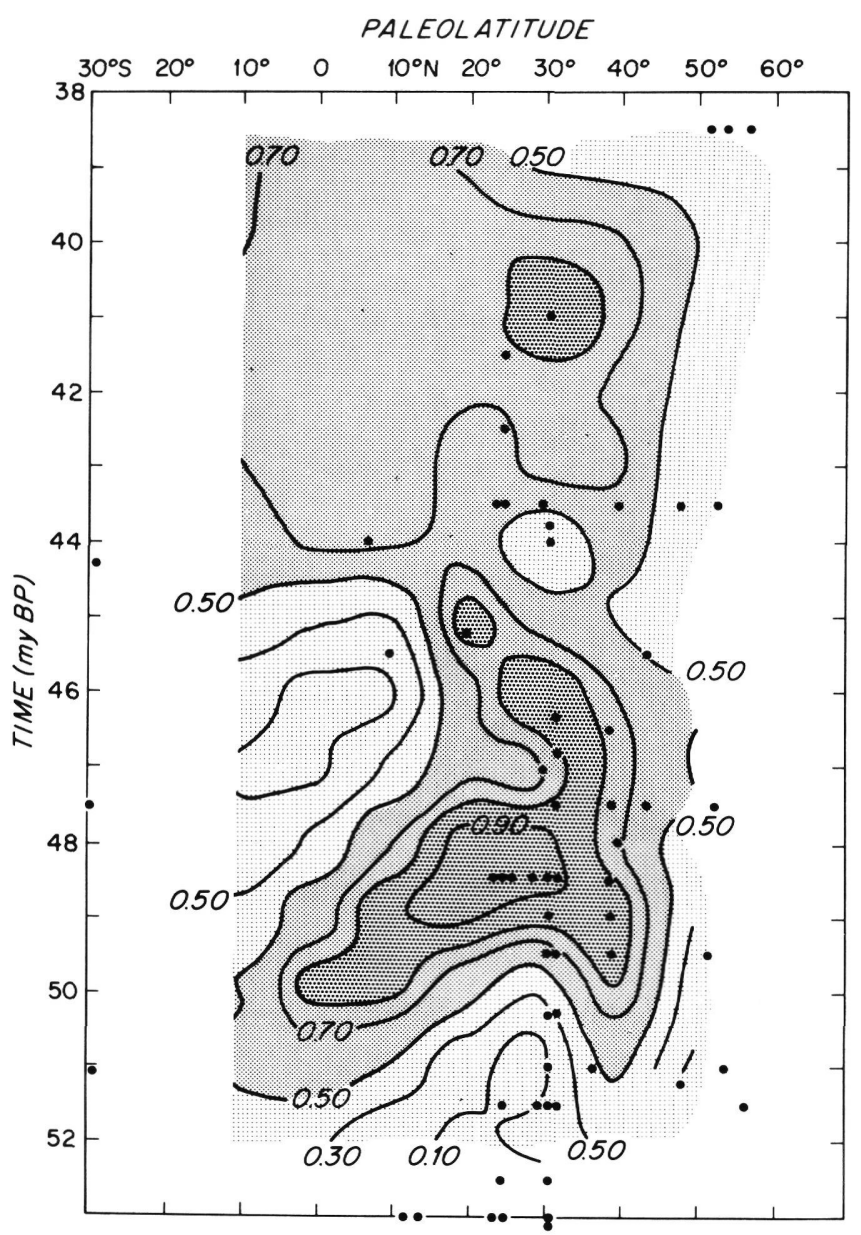

Figure 10. Time and paleolatitudinal distribution of the small to medium sized reticulofenestrids during the Eocene.

tudes is also seen at this time (see Figures 10, 11). (4) A less pronounced warming episode is also evident after 45 m.y.B.P. in the late middle Eocene when discoasters return to mid and lower high latitudes in significant numbers.

\section{CONCLUSIONS}

1. The data presented here show that all three sites of the DSDP Leg 43 considered here contained a nannoflora of intermediate character between the tropical and purely high latitude assemblages of the Atlanticin spite of the position of Site $384\left(41^{\circ} \mathrm{N}\right)$ it contains more lower latitude elements than expected.

2. Site 384 also shows two short-duration blooms: (1) The Cruciplacolithus tenuis-Zygodiscus Assemblage between 64.5 and 63 m.y.B.P., and (2) the Biscutuum? tenuiculum Assemblage at about 62 m.y.B.P. The first bloom is apparently peculiar to this area and has not been observed elsewhere so far. The second is observed in the southern Atlantic during the same short time interval and seems to have been bimodal (see Haq et al., 1977).

3. The biogeographic patterns observed at Leg 43 sites also confirm the earlier paleoclimatic conclusions drawn from the remainder of the North Atlantic bioge-

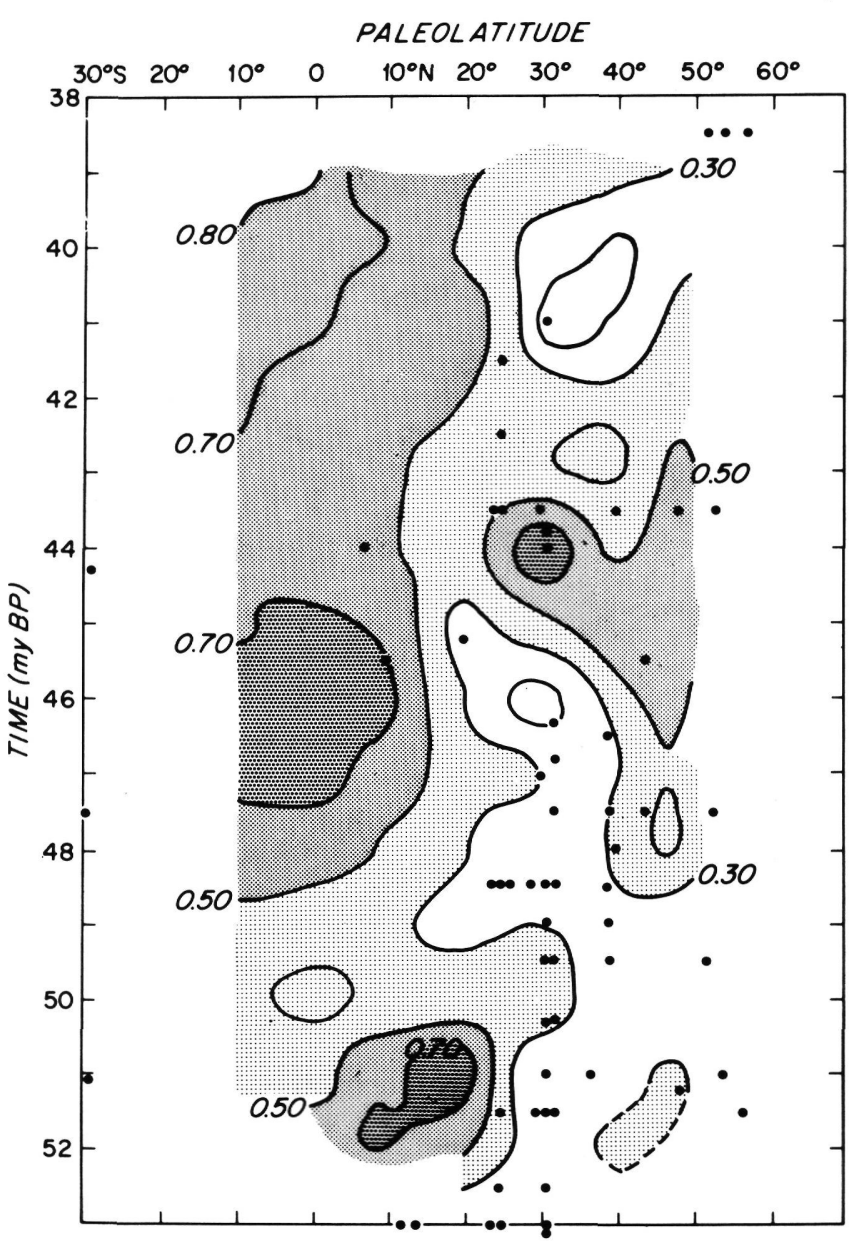

Figure 11. Time and paleolatitudinal distribution of the Discoaster Assemblage during the Eocene.

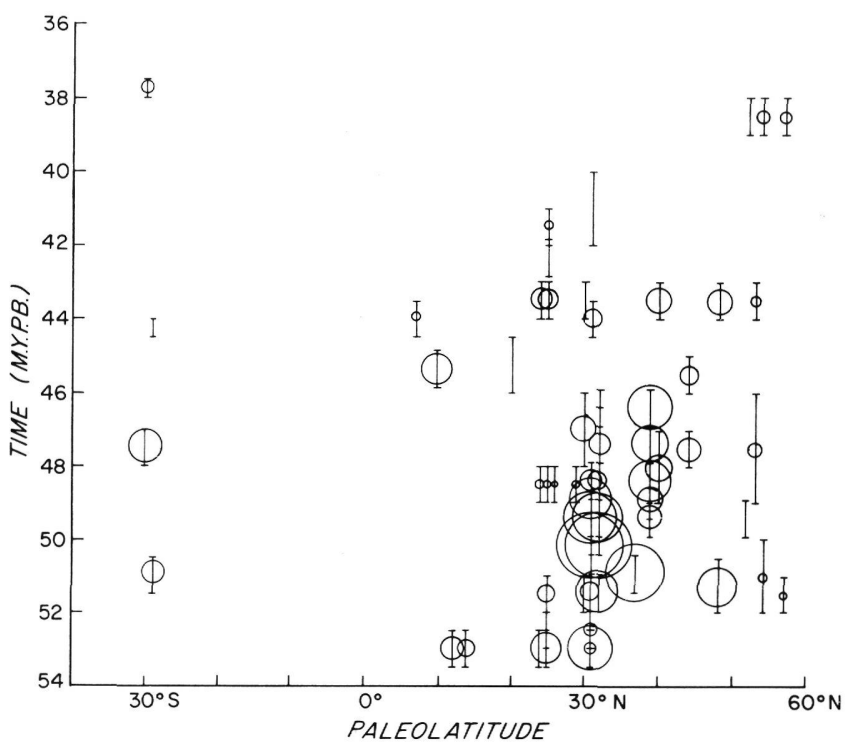

Figure 12. Distribution of the Coccolithus pelagicus Assemblage during the Eocene.

ographic migrationary patterns ( Haq and Lohmann, 1976). In addition, the Leg 43 data help date these 
climatic events more accurately: (1) Marked cooling in middle Paleocene between 61 and 58 m.y.B.P.; (2) marked warming in late Paleocene-early Eocene between 54 and 48 m.y.B.P. with a peak around 50 to 49 m.y.B.P.; (3) cooling in middle Eocene between 48 and 45 m.y.B.P.; and (4) a less pronounced warming in the late middle Eocene between 45 and 43 m.y.B.P.

\section{ACKNOWLEDGMENTS}

This paper was reviewed by W. A. Berggren and R. C. Tjalsma. The research was supported by the Submarine Geology and Geophysics Section of the National Science Foundation, Grants Ga-21983 and GA-2 1274. This is Woods Hole Oceanographic Institution Contribution 3900.

\section{REFERENCES}

Haq, B. U. and Lohmann, G. P., 1976a. Early Cenozoic calcareous nannoplankton biogeography of the Atlantic Ocean: Mar. Micropaleont., v. 1, p. 119-194.
1979. Remarks on the Oligocene calcareous nannoplankton biogeography of the Norwegian Sea (DSDP Leg 38). In Talwani, M., Udintsev, G., et al., Initial Reports of the Deep Sea Drilling Project, Supplement to Volumes 38, 39, 40 and 41: Washington (U.S. Government Printing Office), p. 141-146.

Haq, B. U., Lohmann, G. P., and Wise, S. W., 1977. Calcareous nannoplankton biogeography and its paleoclimatic implications-early Cenozoic of the Falkland Plateau and Miocene of the Atlantic Ocean. In Barker, P., Dalziel, I. W. D., et al., Initial Reports of the Deep Sea Drilling Project, v. 36: Washington (U.S. Government Printing Office), p. 745-760.

Haq, B. U., Perch-Nielsen, K., and Lohmann, G. P., 1977. Contribution to the Paleocene calcareous nannofossil biogeography of the Central and SW Atlantic Ocean (Ceara Rise and São Paulo Plateau, DSDP Leg 39). In PerchNielsen, K., Supko, P. R., et al., Initial Reports of the Deep Sea Drilling Project, v. 39: Washington (U.S. Government Printing Office), p. 841-848. 
PLATE 1

Major and minor components of the Paleocene-early Eocene assemblages. All figures are at same magnification. Scale bar in Figure $10 \mathrm{~b}$ represents $5 \mu \mathrm{m}$.

Figure 1

Figure 2

Figure 5

Figure 6

Figure 7

Figure 8

Figure 9

Figure 10
Prinsius martinii (Perch-Nielsen) Haq. Sample $384-12-1,79-80 \mathrm{~cm}$.

1a. Cross-polarized light.

1b. Phase-contrast.

Prinsius bisulcus (Stradner) Hay and Mohler. Sample 384-11-1, 69-70 cm.

2a. Cross-polarized light.

2b. Phase-contrast.

Coccolithus pelagicus (Wallich) Schiller sensu ampl. Sample 384-11-1, 69-70 cm.

3a. Cross-polarized light.

3b. Phase-contrast.

Ericsonia subpertusa Hay and Mohler. Sample 384-7-6, 109-110 cm.

4a. Cross-polarized light.

4b. Phase-contrast.

Markalius astroporus (Stradner) Hay and Mohler. Sample 384-12-5, $130 \mathrm{~cm}$.

5a. Cross-polarized light.

5b. Phase-contrast.

Toweius craticulus Hay and Mohler. Sample 384-11, CC.

6a. Cross-polarized light.

6b. Phase-contrast.

Thoracosphaera deflandrei Kamptner. Sample 384-10, CC.

7a. Cross-polarized light.

7b. Phase-contrast.

Biscutum ? tenuiculum Okada and Thierstein. Sample 384-12-5, 129-130 cm.

8a. Cross-polarized light.

8 b. Phase-contrast.

Cruciplacolithus tenuis (Stradner) Hay and Mohler. Sample 384-13-2, 99-100 cm.

9a. Cross-polarized light.

9b. Phase-contrast.

Zygodiscus sigmoides Bramlette and Sullivan. Sample 384-11, CC.

10a. Cross-polarized light.

10b. Phase-contrast. 
PLATE 1
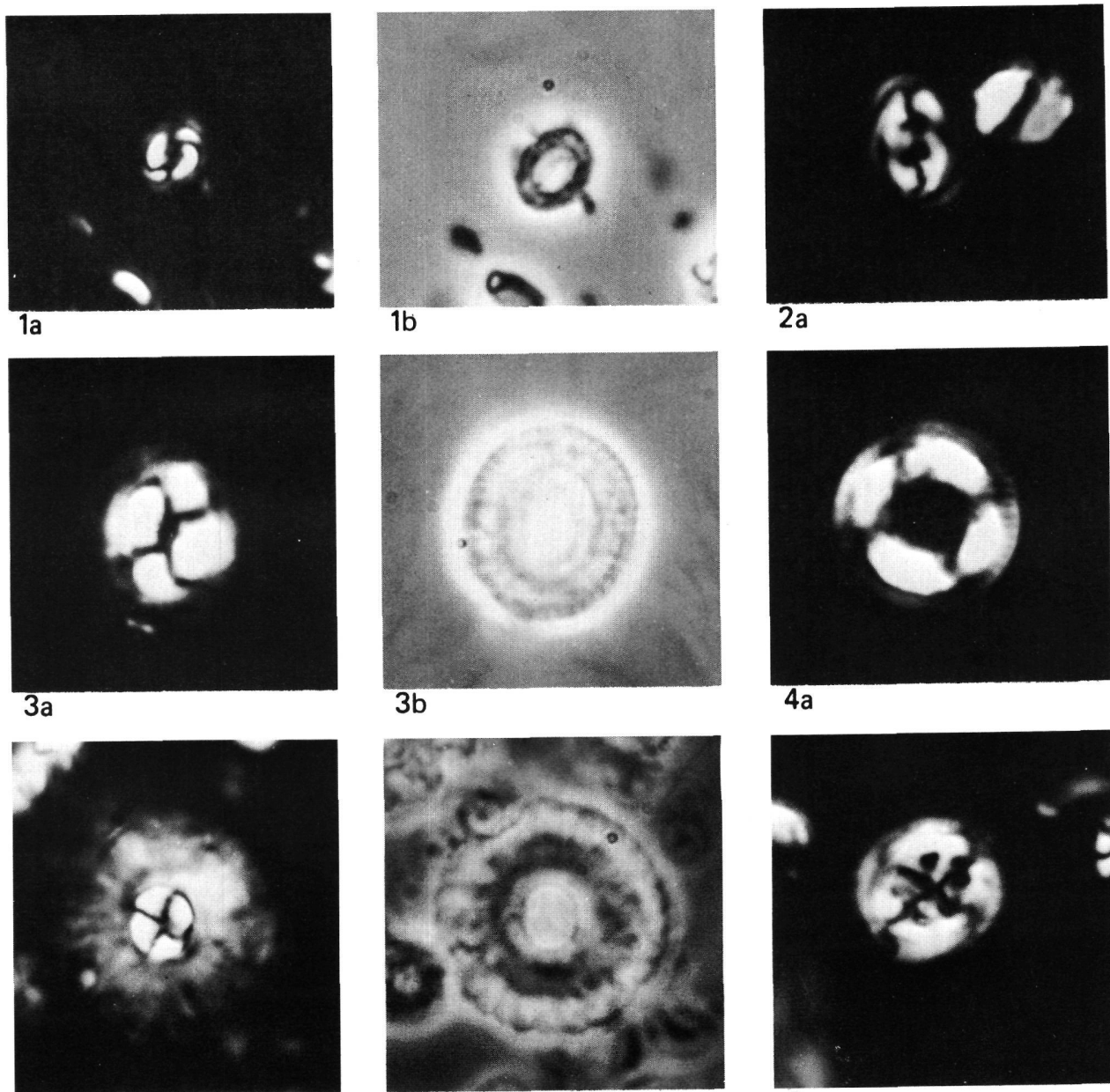

$5 a$

$5 b$

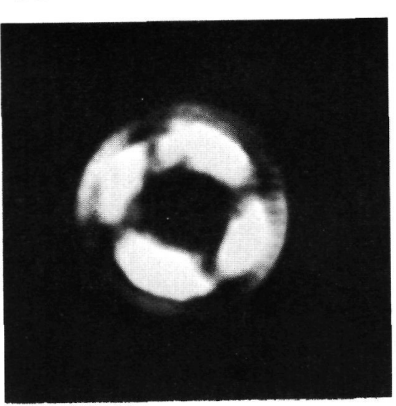

$4 a$

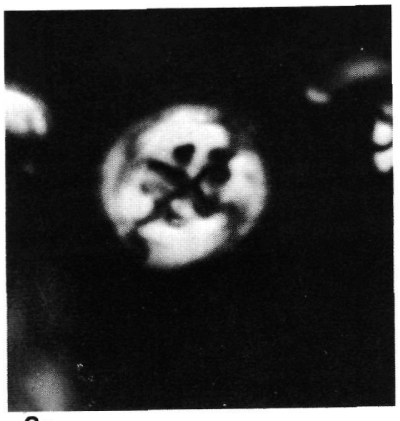

$6 a$

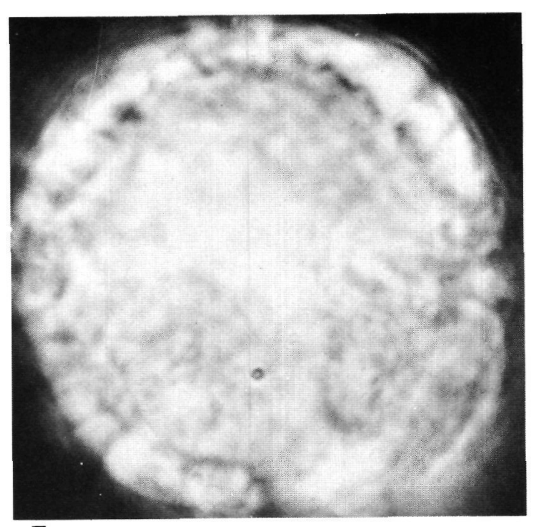

$7 a$

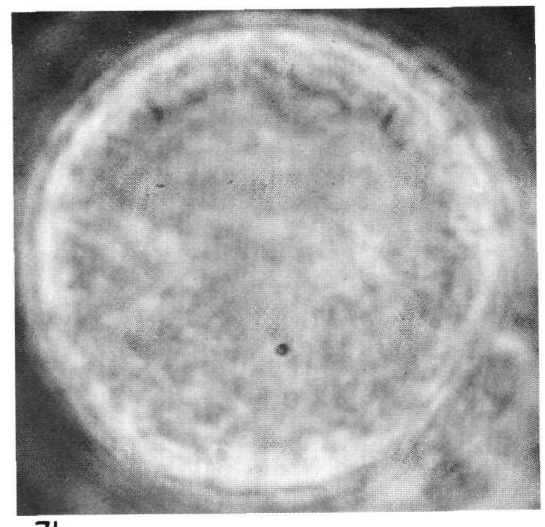

$7 b$

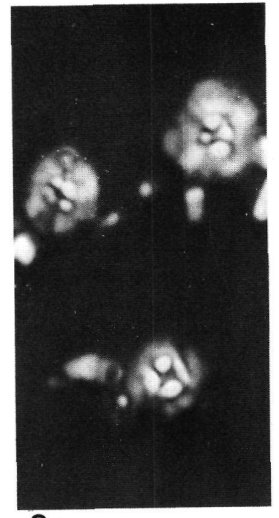

$8 \mathrm{a}$

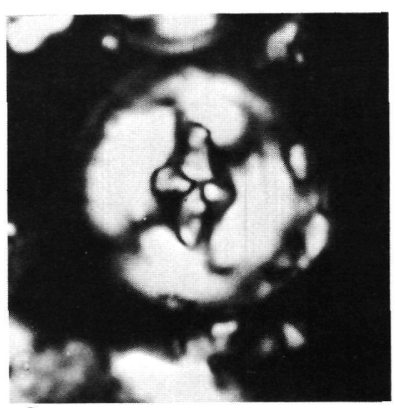

$9 a$

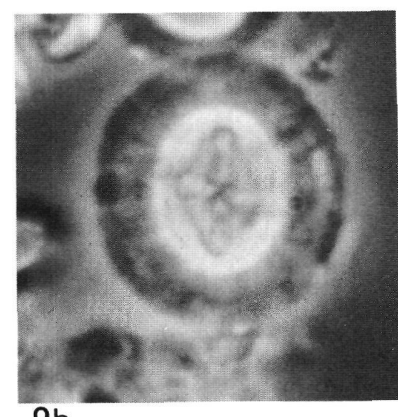

$9 \mathrm{~b}$

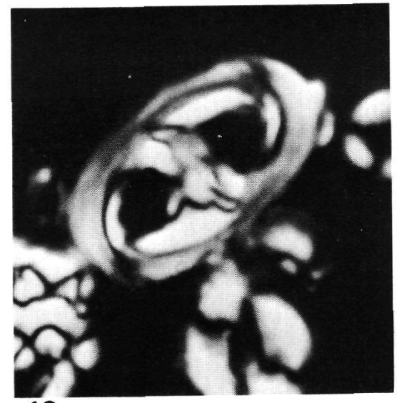

$10 a$

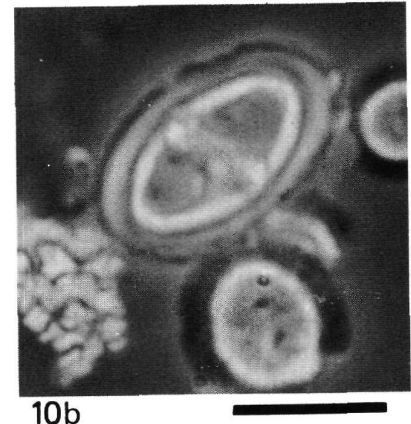


PLATE 2

Major and minor components of the Eocene assemblages. All figures are at same magnification. Scale bar in Figure $10 \mathrm{~b}$ represents $5 \mu \mathrm{m}$.

Figure 1 Discoaster binodosus Martini. Sample 386-33-2, $105-106 \mathrm{~cm}$.

Figure 2 Discoaster diastypus Bramlette and Sullivan. Sample 386-33-2, 105-106 cm.

Figure 3 Discoaster lodoensis Bramlette and Riedel. Sample 384-5-2, 54-55 cm.

Figure 4 Discoaster barbadiensis Tan Sin Hok. Sample $384-5-2,54-55 \mathrm{~cm}$.

Figure 5 Reticulofenestra sp. Sample 384-4-6, 143-144 $\mathrm{cm}$.

5a. Cross-polarized light.

5b. Phase-contrast.

Figure 6 Reticulofenestra dictyoda (Deflandre) Stradner (upper figure) and Reticulofenestra samodurovi (Hay, Mohler and Wade) Roth (lower figure). Sample 384-4-6, 143-144 cm.

6a. Cross-polarized light.

6b. Phase-contrast.

Figure $7 \quad$ Reticulofenestra umbilica (Levin) Martini and Ritzkowski.

7a. Cross-polarized light.

7b. Phase-contrast.

Figure 8 Coccolithus pelagicus (Wallich) Schiller sensu ampl. Sample 384-5-2, $54-55 \mathrm{~cm}$.

8a. Cross-polarized light.

8b. Phase contrast.

Figure 9 Dictyococcites bisectus (Hay, Mohler and Wade) Bukry and Percival.

9a. Cross-polarized light.

9b. Phase-contrast.

Figure 10 Cyclococcolithus formosus Kamptner. Sample $384-5-2,54-55 \mathrm{~cm}$.

10a. Cross-polarized light.

10b. Phase-contrast. 
PLATE 2
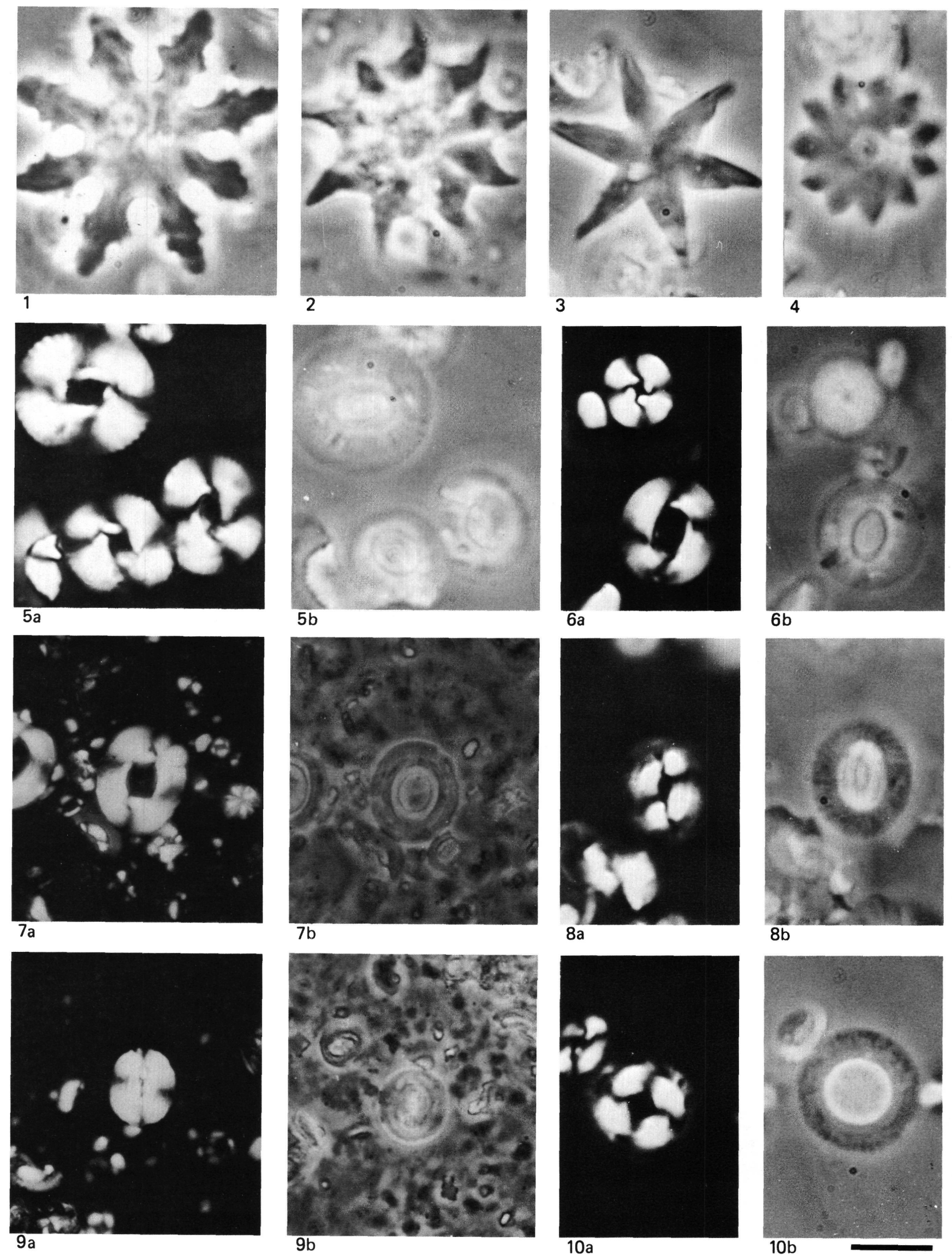